\title{
BMJ Open Mortality in dementia with Lewy bodies compared with Alzheimer's dementia: a retrospective naturalistic cohort study
}

\author{
Annabel Price, ${ }^{1,2}$ Redwan Farooq, ${ }^{3}$ Jin-Min Yuan, ${ }^{3}$ Vandana B Menon, ${ }^{4}$ \\ Rudolf N Cardinal, ${ }^{1,2}$ John T O'Brien ${ }^{1,2}$
}

To cite: Price A, Farooq $R$, Yuan J-M, et al. Mortality in dementia with Lewy bodies compared with Alzheimer's dementia: a retrospective naturalistic cohort study. BMJ Open 2017;7:e017504. doi:10.1136/ bmjopen-2017-017504

- Prepublication history for this paper is available online. To view these files please visit the journal online (http://dx.doi org/10.1136/bmjopen-2017017504).

Received 27 April 2017

Revised 25 August 2017

Accepted 8 September 2017

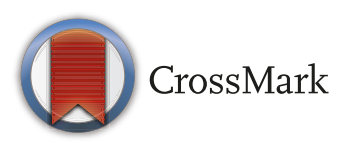

${ }^{1}$ Department of Psychiatry, School of Clinical Medicine, University of Cambridge, Cambridge, UK

${ }^{2}$ Cambridgeshire and Peterborough NHS Foundation Trust, Cambridge, UK ${ }^{3}$ School of Clinical Medicine, University of Cambridge,

London, UK

${ }^{4}$ Norfolk and Suffolk Foundation Trust, Norwich, UK

Correspondence to

Dr Annabel Price;

ap806@medschl.cam.ac.uk

\section{ABSTRACT}

Objectives To use routine clinical data to investigate survival in dementia with Lewy bodies (DLB) compared with Alzheimer's dementia (AD). DLB is the second most common dementia subtype after $A D$, accounting for around $7 \%$ of dementia diagnoses in secondary care, though studies suggest that it is underdiagnosed by up to $50 \%$. Most previous studies of DLB have been based on select research cohorts, so little is known about the outcome of the disease in routine healthcare settings.

Setting Cambridgeshire \& Peterborough NHS Foundation Trust, a mental health trust providing secondary mental health care in England.

Sample 251 DLB and 222 AD identified from an anonymised database derived from electronic clinical case records across an 8-year period (2005-2012), with mortality data updated to May 2015.

Results Raw (uncorrected) median survival was 3.72 years for DLB (95\% Cl 3.33 to 4.14 ) and 6.95 years for $\mathrm{AD}$ (95\% Cl 5.78 to 8.12). Controlling for age at diagnosis, comorbidity and antipsychotic prescribing the model predicted median survival for DLB was 3.3 years $(95 \%$ $\mathrm{Cl} 2.88$ to 3.83$)$ for males and 4.0 years $(95 \% \mathrm{Cl} 3.55$ to 5.00 ) for females, while median survival for $A D$ was 6.7 years $(95 \% \mathrm{Cl} 5.27$ to 8.51$)$ for males and 7.0 years $(95 \%$ Cl 5.92 to 8.73) for females.

Conclusion Survival from first presentation with cognitive impairment was markedly shorter in DLB compared with $A D$, independent of age, sex, physical comorbidity or antipsychotic prescribing. This finding, in one of the largest clinical cohorts of DLB cases assembled to date, adds to existing evidence for poorer survival for DLB versus $A D$. There is an urgent need for further research to understand possible mechanisms accounting for this finding.

\section{INTRODUCTION \\ Background/rationale}

Dementia with Lewy bodies (DLB) accounts for around $7 \%$ of dementia cases in secondary care according to clinic-based prevalence studies, ${ }^{1}$ although other studies have suggested that DLB is underdiagnosed, with up to $50 \%$ of cases missed. ${ }^{2}$

Health services in the UK National Health Service (NHS) comprise primarycare (provided in general practice settings), secondary care

\section{Strengths and limitations of this study}

Large clinical cohort of dementia with Lewy bodies cases.

- Study design reflecting clinical conditions.

- Cases identified by treating clinician diagnosis; therefore, undiagnosed/wrongly diagnosed cases may have been missed.

- Possibility of bias introduced by secondary care study setting.

(specialist care including outpatient services such as memory clinics and inpatient services such as acute psychiatric wards) and tertiary care (subspecialist care provided in selected centres). In UK practice new dementia diagnoses are usually made by clinicians working in secondary care, most commonly old-age psychiatrists and neurologists. There is no clear demarcation between which specialties diagnose which dementia subtypes, and new diagnoses are made in a range of secondary care settings.

Compared with Alzheimer's dementia (AD), studies have suggested that DLB cases have accelerated cognitive decline, more comorbid conditions, a higher mortality rate, greater service use and poorer quality of life. ${ }^{3-8}$ Until recently, it was generally accepted that DLB was more common in males than females, though recent studies have challenged this. ${ }^{1}$

Most previous studies of DLB have been based on select research cohorts, so less is known about the naturalistic patterns, characteristics and outcomes of the disease in routine clinical settings. More recent studies, however, have used dementia registry and population data to examine subtype specific mortality and comorbidity patterns. ${ }^{6-8}$

The emergence of electronic case records and the technology to make these records searchable gives the potential to bring together larger patient cohorts in order to study clinical populations that are otherwise 
difficult to identify. Routine clinical data can now be used to track referral and diagnostic patterns in order to characterise diagnostic trends better and to use these data to inform development of dementia services.

\section{Objectives}

This study aimed to identify a retrospective naturalistic cohort of patients with a diagnosis of DLB within a secondary care sample, describe their demographic and clinical characteristics and measure survival, using as a comparator group a cohort of patients with $\mathrm{AD}$ diagnosed over the same time period.

\section{METHODS}

\section{Study design}

A retrospective cohort design was used. The cohort was identified from the electronic clinical records of Cambridge and Peterborough NHS Foundation Trust (CPFT), which provides secondary mental healthcare to a local population of approximately 900000 people in the UK.

CPFT's electronic records from 2005 to 2012 were deidentified using Case Records Interactive Search software $^{9}$ to create a research database. The deidentification process removes identifying information such as names and addresses from the records and assigns an arbitrary patient-specific research identifier. Such anonymised electronic records methods have been successfully used in secondary mental healthcare to examine variables such as mortality ${ }^{11}$ and incidence of treatment complications. ${ }^{12}$

Data entered onto the system by CPFT clinicians (mental health specialists including doctors, nurses, allied health specialities and social workers) related only to patients currently under the care of secondary mental health services, although they may have been cared for in a number of settings (eg, outpatient clinics, inpatient units and in the community). Some data entered onto the clinical system were recorded in a systematic and structured way (eg, date of birth), while others were recorded as required clinically and in free text (eg, contemporaneous case records, cognitive scores and medical history). Frequency of data entry was guided by clinical necessity and not further specified. The corresponding research database therefore contains some structured data fields (including demographic variables and diagnosis if coded), but the majority of clinical information was found within freetext fields.

\section{Population}

All patients with electronic clinical records in CPFT between 2005 and 2012 (inclusive) were eligible for inclusion in the study. We chose not to include data prior to 2005 for reasons relating to implementation of electronic document storage.

\section{Study sample}

Dementia diagnoses in CPFT were made by psychiatrists specialising in old-age psychiatry.

All patients with a clinician-recorded diagnosis of DLB within this timeframe were included, with a comparator cohort of patients with a clinician-recorded diagnosis of $\mathrm{AD}$ (sampled randomly from all possible such patients; see below).

\section{Case identification}

Key word, phrase and acronym searches based on the diagnosis of DLB (eg, 'Lewy', 'LBD', 'DLB') were applied to the full dataset. Unique document identifiers containing these key words were extracted, with surrounding text containing the key word, phrase or acronym. The same process was repeated for AD. Only records in which the key words or phrases appeared in the initial search were examined further.

An initial manual scan of the extracted text fragments excluded definite non-cases (eg, 'does not have Lewy body dementia'). For the remaining documents, a manual search of the anonymised patient record related to that document was performed.

Manual case identification was then carried out on the records identified by experienced clinicians (AP and VM), with knowledge of diagnostic criteria ${ }^{13} 14$ and symptom presentation in dementia. Cases were positively identified if a diagnosis had been given by a CPFT clinician and it was the most recently recorded diagnosis in the patient record (ie, not later changed to another diagnosis that excluded the diagnosis of interest). Clinician-identified cases were then validated against diagnostic criteria for DLB and AD, respectively. ${ }^{13} 14$

\section{Variables}

Once cases had been positively identified, demographic, clinical and temporal data were extracted from the corresponding anonymised case record. Basic demographic data (eg, date of birth and gender) were extracted automatically using Structured Query Language queries, and clinical data were extracted by clinicians manually by searching the anonymised case records.

Cognitive status was measured using the Mini-Mental State Examination (MMSE). ${ }^{15}$ The MMSE score closest to recorded diagnosis was taken as the MMSE score at diagnosis.

We recorded the date of the first consultation where cognitive impairment was recorded as a problem and the date of diagnosis by month and year.

Physical comorbidy was measured using the Charlson comorbidity index. ${ }^{16}$ This measure contains 19 categories of comorbidity and can be used to predict 10-year mortality for patients who have a range of comorbid conditions. ${ }^{17}$ Each comorbid condition is assigned a score of 1, 2, 3 or 6 depending on the associated mortality risk; for example, metastatic cancer is assigned a score of 6 . The Charlson score has been used in a previous study of comorbidity profile in DLB versus $\mathrm{AD}$, although 
the scores used were not weighted by mortality risk. ${ }^{6}$ We calculated the score that best reflected the physical comorbidities documented in the patient record at the time of diagnosis. All cases were assigned at least a score of 1 due to their dementia diagnosis as per the Charlson scoring algorithm.

Antipsychotic prescribing was recorded as present if any such drugs were documented as being prescribed at any time in the clinical record. Antidementia drug prescribing was recorded as present if the patient had received such a drug (cholinesterase inhibitor or memantine) and continued to take it beyond the initiation phase. Parkinson's disease drug prescribing (dopamine precursor or agonist) was recorded as present if documented at any time in the clinical record.

Mortality data in the database were derived from automatic updates of the source clinical records from the NHS Spine, ${ }^{18}$ including data for patients who were discharged from the service before death. The study end date was May 2015.

\section{Statistical methods}

Baseline demographic and clinical data were analysed using Microsoft's Excel Analysis Toolpak. Within each diagnostic group, we calculated the sex ratio, mean age at diagnosis, mean MMSE at diagnosis, proportions of patients prescribed antipsychotic and antidementia medications and proportions of those with high versus low comorbidity scores on the Charlson index. Continuous variables were compared between the two diagnostic groups using one-way analysis of variance (ANOVA); binary variables were compared using $\chi^{2}$ tests. Results are presented in table 1 .

We analysed survival data using the Cox proportional hazards model, with R V.3.3.0 ${ }^{19}$ and the 'survival' package. We defined each patient's start time as the date (month/year) that they presented with cognitive impairment. If this was not known, then the date of diagnosis was used instead. The end time was either the date of death, or the study end time for surviving patients (May 2015, the dataset's most recent update of NHS spine mortality data). Although dates of diagnosis were not included if before 2005, some records reported date of first presentation with cognitive impairment before 2005.

We tested for baseline differences in potential predictors between the $\mathrm{AD}$ and $\mathrm{DLB}$ groups using one-way ANOVA (for continuous variables) or $\chi^{2}$ tests (for binary variables).

Survival was predicted using discrete factors of diagnosis (AD vs DLB), sex, physical comorbidity (dichotomised as: a 'low' Charlson score of $\leq 2$ vs a 'high' score of $>2$ ) and antipsychotic prescribing at any time (yes/ no). The 'diagnosis' predictor was allowed to interact with each of the other binary predictors (but interactions between sex, comorbidity and antipsychotic prescribing were not included). Age was included as a continuous covariate (not interacting with other predictors). Data are displayed using survival (Kaplan-Meier) plots.

In addition to the full model (IC1 in table 2), we tested a range of simpler models using the following predictors: (C1) diagnosis; (C2) diagnosis, age and sex, with no interactions; (C3) diagnosis, age, sex and frailty, with no interactions; and (C4) diagnosis, age, sex, frailty and antipsychotic prescribing, with no interactions. We compared sequential models using likelihood ratio tests to see if the addition of additional predictors was justified. We also tested an equivalent set of models (D1-D4 and ID1) using the time since diagnosis as the dependent variable, rather than the time since presentation with cognitive impairment, to see if the same pattern of results held.

\begin{tabular}{|c|c|c|c|c|}
\hline Characteristic & DLB $(n=251)$ & $A D(n=222)$ & $\chi^{2}$ or $F$ test statistic & $\mathrm{p}$ Value \\
\hline \multicolumn{5}{|l|}{ Gender } \\
\hline Female & $129(51.4 \%)$ & $139(62.6 \%)$ & $\chi_{1}^{2}=6.04$ & 0.01 \\
\hline Male & 122 & 83 & & \\
\hline $\begin{array}{l}\text { Age at first presentation with cognitive } \\
\text { impairment (years) }\end{array}$ & $78.8(\mathrm{SD} 7.7)(\mathrm{n}=251)$ & $79.5(S D 8.8)(n=200)$ & $F_{1,449}=2.51$ & 0.11 \\
\hline Age at diagnosis (years) & 79.3 (SD 7.6) & 80.2 (SD 8.8) & $F_{1,471}=1.32$ & 0.25 \\
\hline MMSE score at diagnosis & $20.1(\mathrm{SD} 5.5)(\mathrm{n}=183)$ & $20.6(\mathrm{SD} 4.9)(\mathrm{n}=172)$ & $F_{1,353}<1$ & 0.41 \\
\hline \multicolumn{5}{|l|}{ Charlson comorbidity index at diagnosis } \\
\hline Low comorbidity (score $\leq 2$ ) & $183(72.9 \%)$ & $158(71.2 \%)$ & $\chi_{1}^{2}=0.18$ & 0.67 \\
\hline High comorbidity (score >2) & 68 & 64 & & \\
\hline \multicolumn{5}{|l|}{ Medications prescribed } \\
\hline Antipsychotic (neuroleptic) drugs & $103(41.0 \%)$ & $32(14.4 \%)$ & $\chi_{1}^{2}=39.6$ & $<0.0001$ \\
\hline Parkinson's disease drugs & $93(37.1 \%)$ & $0(0 \%)$ & $\chi^{2}{ }_{1}=102.39$ & $<0.0001$ \\
\hline Antidementia drugs & $152(60.6 \%)$ & $139(62.6 \%)$ & $\chi_{1}=0.21$ & 0.65 \\
\hline
\end{tabular}

AD, Alzheimer's disease; DLB, dementia with Lewy bodies; MMSE, Mini-Mental State Examination. 


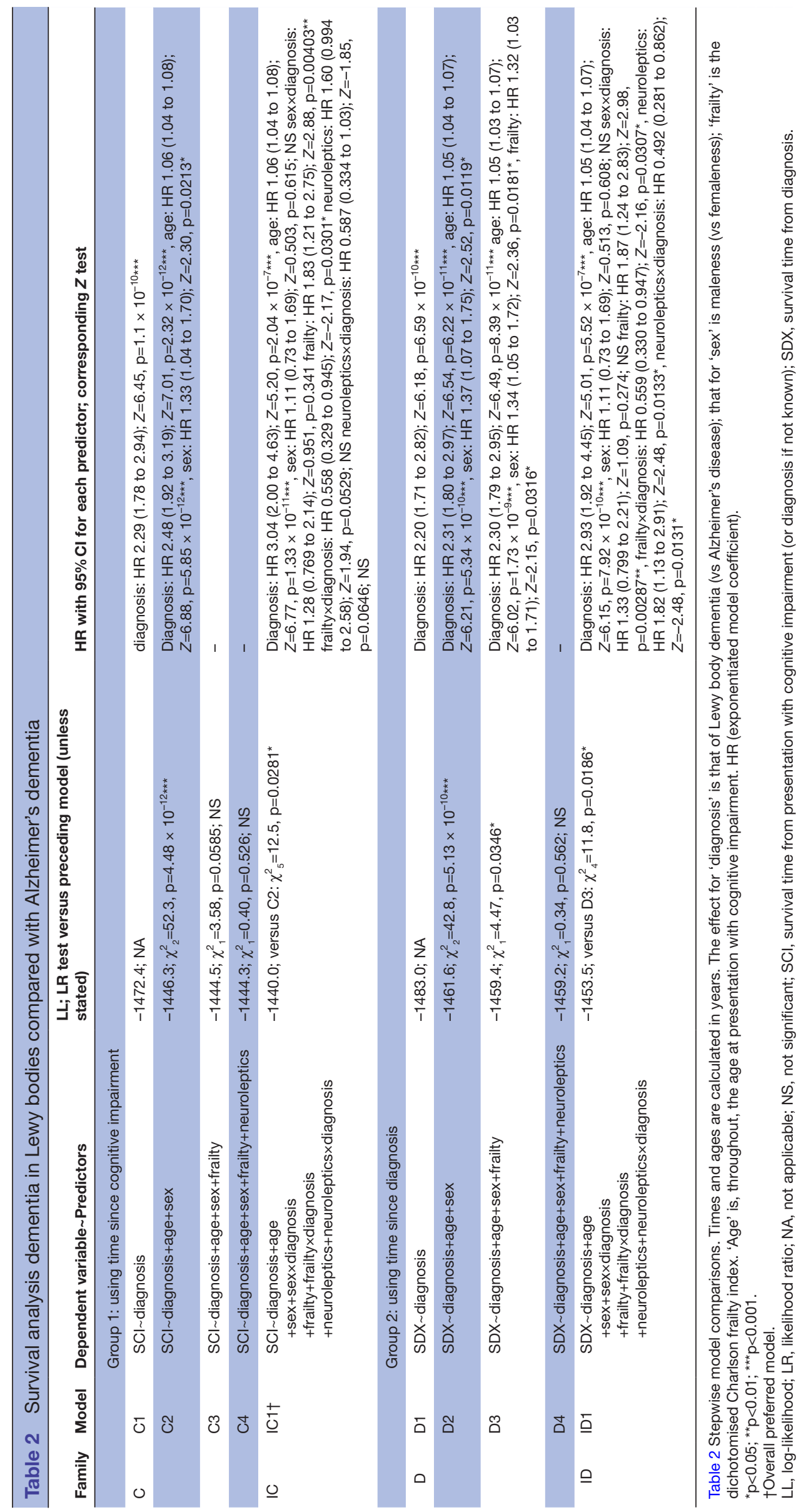




\section{Patient involvement}

Patients were not involved directly in this study, and patient level data were not identifiable due to the anonymisation process. The authors worked closely with a CPFT dementia patient and carer advisory group who advised on research priorities and agenda setting during the project.

\section{RESULTS}

\section{Sample}

The initial text word search in the DLB case identification process across the entire time period of the database yielded 2276 separate clinical documents (eg, clinic letters) pertaining to 983 unique patient records. Manual searching of these records to exclude non-cases yielded a total of 304 individual cases in the database. Over the 8-year study period (2005-2012), there were 251 new diagnoses of DLB made within CPFT.

For the AD cohort, the initial text search yielded 21424 unique clinical documents pertaining to 7442 unique patient records. If a similar case-finding ratio is assumed, then there would be approximately 2304 cases of $\mathrm{AD}$ in the database in total. Data were gathered for 254 randomly selected cases of $\mathrm{AD}$ for comparison (approximately $10 \%$ of expected total cases). Of these, 222 were newly diagnosed between 2005 and 2012, and these were used as the comparator group.

\section{Main results}

In the $\mathrm{AD}$ cohort, $153(69 \%)$ patients had been given a diagnosis of dementia in Alzheimer's disease, 66 (30\%) had been given a diagnosis of atypical or mixed AD and $3(1 \%)$ had been given a diagnosis of $\mathrm{AD}$ with early onset. In the DLB cohort, all the patients had been given a diagnosis of DLB (rather than a dementia in Parkinson's disease or mixed DLB) diagnosis. We validated the clinician-diagnosed DLB cases against standard diagnostic criteria and found that 244/251 (97\%) had probable or possible DLB, and of those, $58 \%$ were probable and $39 \%$ were possible. All of the patients in the AD cohort met the diagnostic criteria for AD.

In the DLB cohort, there was an overall year-onyear increase in new diagnoses across the 8-year study period. An upwards trend in annual diagnoses was also found in the AD group.

There were no differences between the DLB and $\mathrm{AD}$ groups in mean age at presentation with cognitive impairment or diagnosis, mean MMSE at diagnosis, physical comorbidity burden at diagnosis or antidementia drug prescribing. There was, however, a significantly higher ratio of females to males in the $\mathrm{AD}$ compared with the DLB group. There were also significant differences between groups in antipsychotic prescribing and Parkinson's drug prescribing, both being higher in the DLB group (see table 1 ).

Age at diagnosis did not differ significantly between groups (mean $\pm \mathrm{SD}$ : AD: $80.2 \pm 8.8$ years, $n=222$; DLB:
$79.3 \pm 7.6$ years, $\mathrm{n}=251$ ) (one-way ANOVA, $F_{1,471}=1.32$, $\mathrm{p}=0.25)$. Age residuals deviated from a normal distribution (Shapiro-Wilk test, $\mathrm{W}=0.968, \mathrm{p}=1.33 \times 10^{-8}$ ), and a quantile-quantile $(\mathrm{Q}-\mathrm{Q})$ plot showed that age exhibited some minor negative skew and was somewhat leptokurtic; ANOVA is robust to this situation. ${ }^{20}$

MMSE at diagnosis did not differ significantly between groups (mean \pm SD: AD: 20.6 $\pm 4.9, \mathrm{n}=172$; DLB 20.1 \pm 5.5 , n=183) (one-way ANOVA, $F<1$, NS). MMSE residuals deviated from a normal distribution (Shapiro-Wilk test, $\mathrm{W}=0.967, \mathrm{p}=3.27 \times 10^{-7}$ ), and a $\mathrm{Q}-\mathrm{Q}$ plot showed that MMSE also exhibited some minor negative skew, although the distribution was very close to mesokurtic (Pearson kurtosis 3.2); again, ANOVA is robust to this situation. $^{20}$

Age at presentation with cognitive impairment was available for all patients with DLB and 200/222 patients with $\mathrm{AD}$. There were no group differences (see table 1). As for age at diagnosis, residuals for age at cognitive impairment deviated from a normal distribution (Shapiro-Wilk $\mathrm{W}=0.969, \mathrm{p}<0.001)$ by being slightly negatively skewed (skew -0.77) and leptokurtic (Pearson kurtosis 4.29 ) to which ANOVA is robust. ${ }^{20}$ In the survival analysis below, 'age at presentation with cognitive impairment' is replaced with 'age at diagnosis' for those subjects for whom age at presentation with cognitive impairment was unavailable (see Methods).

\section{Survival analysis}

Median survival for DLB was significantly shorter in the DLB group compared with the AD group. Raw (uncorrected) median survival was 3.72 years for DLB $(95 \%$ CI 3.33 to 4.14 ) and 6.95 years for $\mathrm{AD}(95 \% \mathrm{CI} 5.78$ to $8.12)$. For males, median survival in DLB was 3.57 years (95\% CI 3.24 to 4.14 ) and in AD 6.46 years $(95 \%$ CI 5.42 to 8.12 ). For females median survival in DLB was 3.81 years (95\% CI 3.36 to 4.50 ) and in $\mathrm{AD} 7.03$ years (95\% CI 5.92 to 8.73$)$.

The best fit model for survival from date of presentation with cognitive impairment included diagnosis, age, sex, frailty and neuroleptic prescribing with sex and diagnosis, frailty and diagnosis and neuroleptic prescribing and diagnosis included as interacting terms (see table 2). The difference in survival from time of presentation with cognitive impairment was not explained by any differences in sex, age, comorbidity burden or antipsychotic prescribing. In the overall model, there was a large effect of diagnosis (HR 3.04 for $\mathrm{DLB}$ vs $\mathrm{AD}, Z=5.2, \mathrm{p}<0.001)$. As expected, there was an effect of age (HR of 1.06 for every year older; $Z=6.77$, $\mathrm{p}<0.001$ ), although ages were not different between the diagnostic groups (table 1) and the effect of diagnosis was found over and above the effect of age.

There was no main effect of $\operatorname{sex}(Z=0.50, \mathrm{NS})$ and no interaction between diagnosis and $\operatorname{sex}(Z=0.95$, NS). There was an effect of comorbidity that interacted with diagnosis $(Z=-2.17, \mathrm{p}=0.030)$, but this effect was only seen in the $\mathrm{AD}$ group (subanalysis for $\mathrm{AD}$ with 


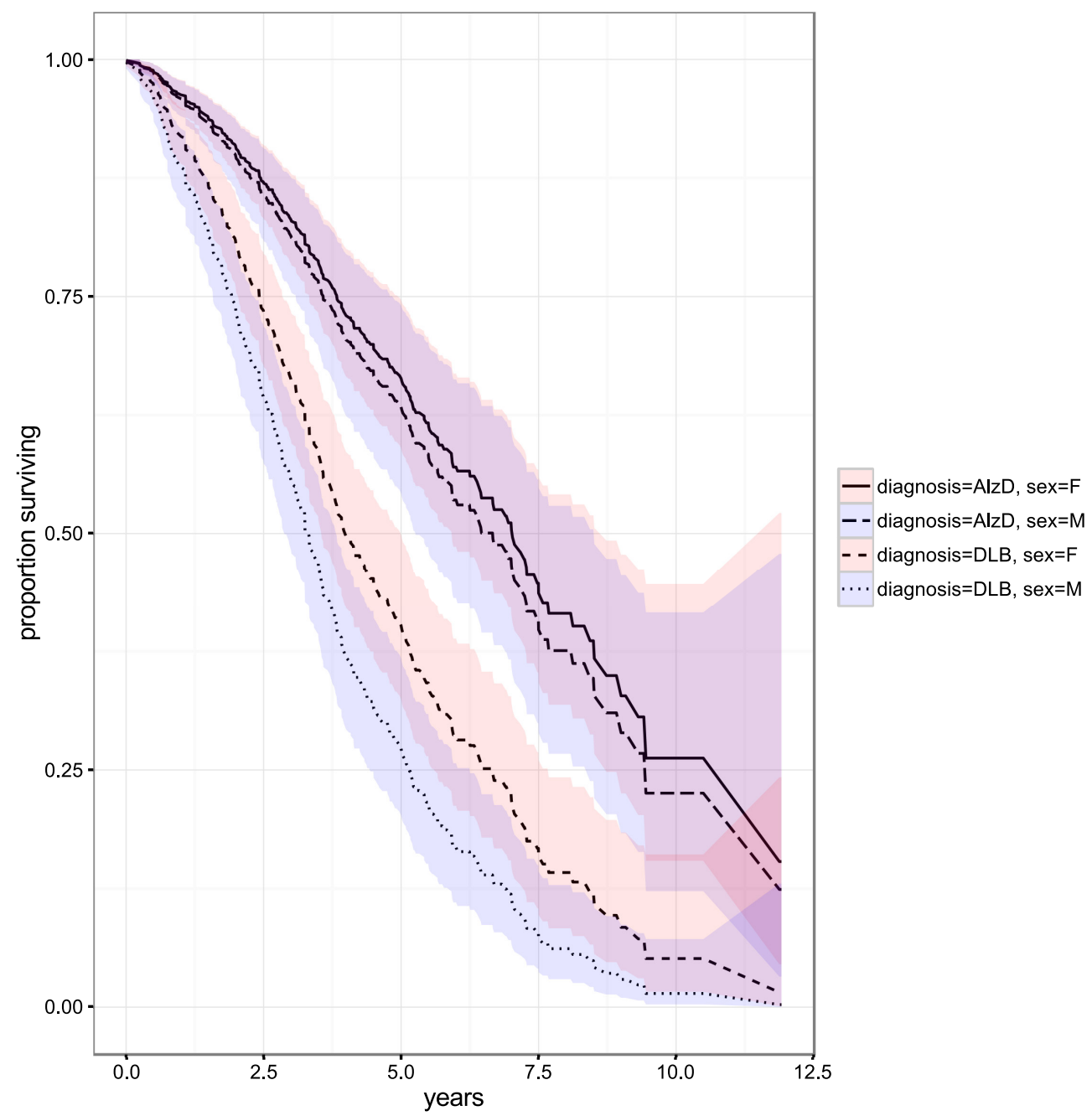

Figure 1 Survival in dementia with Lewy bodies (DLB) versus Alzheimer's dementia (AlzD). Bands indicate 95\% Cls for the cumulative hazard. F, female; M, male.

sex, antipsychotic prescribing, comorbidity and age as predictors: effect of comorbidity, HR $1.82, Z=2.86$, $\mathrm{p}=0.004$ ) and not in the DLB group (similar analysis; $Z=0.18$, NS). The effect of antipsychotic prescribing did not reach significance, either as a main effect (HR $1.60, Z=1.94, p=0.053)$ or as an interaction with diagnosis $(Z=-1.85, \mathrm{p}=0.065)$, although the trend was for a numerically greater adverse effect of antipsychotics in $\mathrm{AD}$ (subgroup analysis as before, $\mathrm{HR}=1.60, Z=1.92$, $\mathrm{p}=0.055)$ than in DLB $(\mathrm{HR}=0.94, Z=-0.37, \mathrm{p}=0.71)$.

Survival by diagnosis and sex is presented in figure 1 .

At the global mean values for age at diagnosis (79.4 years), comorbidity (dichotomised frailty index 0.279 ) and antipsychotic prescribing (0.285), the model predicted median survival for DLB was 3.3 years (95\% CI 2.88 to 3.83 ) for males and 4.0 years $(95 \%$ CI 3.55 to 5.00) for females, while median survival for AD was 6.7 years (95\% CI 5.27 to 8.51 ) for males and 7.0 years (95\% CI 5.92 to 8.73) for females.

In order to ascertain if there was any bias introduced by choosing the start time as date of presentation with cognitive impairment rather than date of diagnosis, we repeated the survival analysis using date of diagnosis as the start time. We found few differences between the results of the two analyses (see table 2).

\section{DISCUSSION}

\section{Key results}

Survival was markedly poorer for the DLB cohort than the AD cohort. This difference was not explained by sex, stage of dementia or age at presentation, comorbidity burden or drug prescribing. Our study showed a differential survival between AD and DLB of around 3 years for both males and females with survival being shorter in males in both cohorts. In our study, men with DLB had the poorest survival numerically, followed by women with DLB, men with $\mathrm{AD}$ then women with $\mathrm{AD}$; however, the sex differences were not significant in the full adjusted model (table 2). In contrast, a previous study ${ }^{3}$ found women with DLB to have the poorest survival, followed by men with DLB, men with $\mathrm{AD}$, then women with AD. Previous studies in DLB have shown survival of 5.5-7.7 years from disease 
onset and 1.9-6.3 years from diagnosis. ${ }^{7}$ Our findings are therefore in line with those from other mortality studies in DLB as the time of first presentation would be expected to be between symptom onset and diagnosis. Recent national data on dementia mortality in the Mental Health Minimum Data Set (MHMDS) shows a median survival of 3.5 years from diagnosis with cognitive impairment or dementia at 'moderate need' (not further defined) with shorter survival times when needs are high or very high. ${ }^{21}$ This is somewhat surprising given that the mortality of the lowest needs group reported in the MHMDS sample is in line with the poorer surviving of our two cohorts. The MHMDS does not report the diagnostic breakdown of the population studied nor does it report any adjustment for other potential confounders.

We did not find a significant association between antipsychotic prescribing and mortality, which was surprising especially in the DLB cohort where there is well-characterised sensitivity to antipsychotic medication. ${ }^{22}$

Comorbidity burden was specifically associated with mortality in the $\mathrm{AD}$ group only, which was surprising given that other studies have found that increased comorbidity burden is associated with increased risk of mortality in dementia including DLB in both a research cohort ${ }^{3}$ and a whole population epidemiological study. ${ }^{8}$ In our study, we used a dichotomised comorbidy index of 'low' versus 'high' rather than the mean Charlson score, more detailed symptom profiles ${ }^{36}$ or number of prescribed medications. ${ }^{8}$ Where finer grained physical comorbidity data have been included in analyses, resting tremor was the only single physical comorbidity related to survival and was found to be a protective factor. ${ }^{3}$ When comorbidity profiles have been compared between DLB and $\mathrm{AD}$ cohorts, overall comorbidity burden has been shown to be higher in DLB, from the time of diagnosis, with increased cerebrovascular comorbidity. ${ }^{6}$ In contrast, we found that at the time of diagnosis, there was little difference between the two groups (though we were not able gather data to the same level of detail).

This study further strengthens the findings from a number of studies that survival is poorer in DLB than AD, although in our cohort, this finding was not accounted for by other factors measured, including physical comorbidity burden, suggesting that there may be an intrinsically higher rate of mortality in DLB than AD.

Further work is needed both to examine factors associated with this excess mortality in DLB in this and other cohorts, so that high-risk subjects can be identified, and to elucidate potential mechanisms that may underpin this increase to inform intervention studies. A number of potential factors have been identified, although often from small studies. These include clinical features such as the presence of autonomic symptoms and hallucinations, and pathological features such as increased cerebrospinal fluid tau and apolipoprotein $\mathrm{E} 4 .^{7}$

\section{Strengths}

Clinical information was extracted from the electronic case records by experienced clinicians, to a clear protocol, and validated against accepted diagnostic criteria. The advantage of identifying a large retrospective sample is that the characteristics of the sample are reflective of a clinical population. The identification of a cohort of $\mathrm{AD}$ cases in the same service during the same time frame allowed for comparisons to be made under similar clinical conditions.

\section{Limitations}

This study focused on identification of two clinically diagnosed dementia subtypes, DLB and a comparison group with $\mathrm{AD}$, with a complete cohort of DLB cases identified over an 8-year period. Cases were primarily identified as $\mathrm{DLB}$ or $\mathrm{AD}$ in the study if they were assigned the diagnosis by the treating clinician and then validated against diagnostic criteria. It is possible, though, that a small number of cases were misclassified and, if a prospective case identification strategy had been used, these cases would have been assigned a different dementia subtype diagnosis. Low diagnostic accuracy ${ }^{23}$ and comorbidity between DLB and AD in dementia ${ }^{24}$ has previously been shown in pathological DLB cohorts. We were also not able to extract consistent data on the temporal onset of core DLB features. It is therefore possible that a subset of patients with more advanced $\mathrm{AD}$ were misclassified as having DLB based on the core symptom profile. Using our methodological approach, we were not able to identify patients who would have fulfilled criteria for DLB but had not been diagnosed with the condition as our search strategy relied on text terms associated with the diagnostic descriptions. Given the limitations above, the difference between the two clinically diagnosed cohorts regarding survival is therefore more striking as one might expect the differences to be less marked given lower diagnostic accuracy in a clinical rather than pathologically diagnosed study sample.

The case note design meant that while we were able to capture data from a naturalistic cohort accessing secondary care, we were not able to ensure the completeness of data that might be acquired in a research sample. In particular, we extracted the comorbidity data only from what we were able to gather from the patient record and we did not have a complete set of MMSE scores at diagnosis. In addition, we used MMSE as our primary measure of cognitive function, as this was the tool routinely used in clinical practice, but this would not have given a complete picture of cognitive dysfunction particularly in the DLB cohort.

The cohorts analysed in this study were selected based on their diagnoses being made within a specified time frame and were not more specifically matched, although every attempt was made to minimise bias in identification of the comparator cohort, and subsequent analysis found few significant differences in demographic and clinical characteristics. It is possible, though, that the 
study outcome would have been different if a more robust matching strategy had been employed.

The study sample comprised two diagnostic groups over a specified time period in a secondary mental healthcare setting. It is possible that the findings of the study do not reflect the total populations with these diagnoses. Diagnosis in a secondary care setting may reflect greater symptom severity, for example, although in the UK, the great majority of new diagnoses of dementia (and subsequent initiation of treatment) are currently made in secondary care following GP or specialist referral. ${ }^{25}$ The mean MMSE at diagnosis for both groups was in the mildto-moderate range of severity of cognitive impairment, with similar SD. The study findings may be limited by not identifying patients at earlier stages of disease, although patients referred into secondary care (especially to community mental health teams) are likely to be referred with functional decline or other related difficulty, which will usually occur beyond the earliest stages of disease. Previous studies of mortality in DLB have reported similar baseline mean MMSE scores. ${ }^{6} 8$

The retrospective nature of the study meant that accurate estimation of timing of symptom onset was not possible, limiting our ability to report duration of illness accurately. To minimise any bias introduced by differential timing of diagnoses between the groups (although there was no evidence in the baseline data to suggest this was the case), we based the primary survival analysis on the date of first presentation with cognitive impairment, rather than the date of diagnosis, but reanalysis using date of diagnosis produced similar results.

\section{Interpretation}

The underlying reason for the difference in mortality rates between the DLB and AD cohorts remains unclear, but this study adds to the existing evidence showing a higher mortality rate for DLB than AD, although not accounted for by other factors including comorbidity burden.

\section{Generalisability}

CPFT is a relatively small mental health trust, but because the methodology used identified a naturalistic clinical sample, it is likely that the cohorts identified are representative of a wider secondary care population with dementia. A number of other mental health trusts have or are developing the capability to use anonymised clinical records for research. The methodology used to select the cohorts for this study could be repeated on these trusts' clinical records and findings compared with determine whether our study's findings generalise across other clinical populations.

It was not possible to gather consistent or complete data on ethnicity, education level and level of deprivation. If we had been able to gather these data, it may have been possible to discuss in more detail how the results of this study may be comparable with other dementia populations. Considering the CPFT patient population as a whole, however, the Trust serves a fast-growing, ageing and diverse population with significant inequalities, ${ }^{26}$ which are likely to be reflected in our study cohort.

Acknowledgements We thank the patient and carer advisory group that have supported the authors throughout this study and beyond and Jonathan Lewis, CPFT Research Database Manager, for his invaluable support and the Alzheimer's Society for supporting this research.

Contributors $\mathrm{JO}$ had the original idea for the study, and AP and J0 designed the study. AP, RF, J-MY and VM completed case identification and data extraction, and $A P$ and $R C$ analysed the data. AP drafted the manuscript, and all authors contributed to the finished manuscript. AP is guarantor.

Funding The CPFT Research Database was supported by the UK National Institute of Health Research Cambridge Biomedical Research Unit in Dementia and the Biomedical Research Centre, and Cambridgeshire and Peterborough NHS Foundation Trust. AP was funded by the National Institute of Health Research's (NIHR) Collaboration for Leadership in applied Health Research and Care East of England (CLAHRC EoE) Fellowship programme. The researchers were independent of the above funders.

Competing interests None declared.

Ethics approval Ethical approval for this process was granted by the UK NHS National Research Ethics Service reference 12/EE/0407. There was also projectspecific NHS Institutional review.

Provenance and peer review Not commissioned; externally peer reviewed.

Data sharing statement Full dataset and statistical code available from the corresponding author. Individual consent was not obtained, but the presented data are anonymised and aggregated.

Open Access This is an Open Access article distributed in accordance with the Creative Commons Attribution Non Commercial (CC BY-NC 4.0) license, which permits others to distribute, remix, adapt, build upon this work non-commercially, and license their derivative works on different terms, provided the original work is properly cited and the use is non-commercial. See: http://creativecommons.org/ licenses/by-nc/4.0/

(c) Article author(s) (or their employer(s) unless otherwise stated in the text of the article) 2017. All rights reserved. No commercial use is permitted unless otherwise expressly granted.

\section{REFERENCES}

1. Vann Jones SA, O'Brien JT. The prevalence and incidence of dementia with Lewy bodies: a systematic review of population and clinical studies. Psychol Med 2014;44:673-83.

2. Palmqvist $S$, Hansson $O$, Minthon $L$, et al. Practical suggestions on how to differentiate dementia with Lewy bodies from Alzheimer's disease with common cognitive tests. Int J Geriatr Psychiatry 2009;24:1405-12.

3. Williams MM, Xiong C, Morris JC, et al. Survival and mortality differences between dementia with Lewy bodies vs Alzheimer disease. Neurology 2006;67:1935-41.

4. Boström F, Jönsson L, Minthon L, et al. Patients with Lewy body dementia use more resources than those with Alzheimer's disease. Int J Geriatr Psychiatry 2007;22:713-9.

5. Boström F, Jönsson L, Minthon L, et al. Patients with dementia with lewy bodies have more impaired quality of life than patients with Alzheimer disease. Alzheimer Dis Assoc Disord 2007;21:150-4.

6. Fereshtehnejad SM, Damangir S, Cermakova P, et al. Comorbidity profile in dementia with Lewy bodies versus Alzheimer's disease: a linkage study between the Swedish Dementia Registry and the Swedish National Patient Registry. Alzheimers Res Ther 2014;6:65.

7. Mueller C, Ballard C, Corbett A, et al. The prognosis of dementia with Lewy bodies. Lancet Neurol 2017;16:390-8.

8. Garcia-Ptacek S, Farahmand B, Kåreholt I, et al. Mortality risk after dementia diagnosis by dementia type and underlying factors: a cohort of 15,209 patients based on the Swedish Dementia Registry. J Alzheimers Dis 2014;41:467-77.

9. Fernandes AC, Cloete D, Broadbent MT, et al. Development and evaluation of a de-identification procedure for a case register sourced from mental health electronic records. BMC Med Inform Decis Mak 2013;13:71.

10. Chang CK, Hayes RD, Perera G, et al. Life expectancy at birth for people with serious mental illness and other major disorders from 
a secondary mental health care case register in London. PLoS One 2011;6:e19590.

11. Fok ML, Hayes RD, Chang CK, et al. Life expectancy at birth and all-cause mortality among people with personality disorder. J Psychosom Res 2012;73:104-7.

12. Chang CK, Harrison S, Lee W, et al. Ascertaining instances of neuroleptic malignant syndrome in a secondary mental healthcare electronic medical records database: the SLAM BRC Case Register. Ther Adv Psychopharmacol 2012;2:75-83.

13. McKeith IG, Dickson DW, Lowe J, et al. Diagnosis and management of dementia with Lewy bodies: third report of the DLB Consortium. Neurology 2005;65:1863-72.

14. McKhann GM, Knopman DS, Chertkow $\mathrm{H}$, et al. The diagnosis of dementia due to Alzheimer's disease: recommendations from the National Institute on Aging-Alzheimer's Association workgroups on diagnostic guidelines for Alzheimer's disease. Alzheimers Dement 2011;7:263-9.

15. Folstein MF, Folstein SE, McHugh PR. "Mini-mental state". A practical method for grading the cognitive state of patients for the clinician. J Psychiatr Res 1975;12:189-98.

16. Charlson ME, Pompei P, Ales KL, et al. A new method of classifying prognostic comorbidity in longitudinal studies: development and validation. J Chronic Dis 1987;40:373-83.

17. Charlson M, Szatrowski TP, Peterson J, et al. Validation of a combined comorbidity index. J Clin Epidemiol 1994;47:1245-51.
18. Health and Social Care Information Centre ( $\mathrm{HSClC})$ Spine Services. http://systems.hscic.gov.uk/spine

19. Core Team R. A language and environment for statistical computing Vienna. Austria, 2016. https://www.R-project.org/

20. Myers JL, Well AD. Research design and statistical analysis. Hillsdale, New Jersey: Lawrence Erlbaum Associates, 1995.

21. Health and Social Care Information Centre. Focus on dementia, 2016. http://content.digital.nhs.uk/catalogue/PUB19812/Focus-ondementia-jan-2016-v1-r1.pdf

22. Ballard C, Grace J, McKeith I, et al. Neuroleptic sensitivity in dementia with Lewy bodies and Alzheimer's disease. Lancet 1998;351:1032-3.

23. Nelson PT, Jicha GA, Kryscio RJ, et al. Low sensitivity in clinical diagnoses of dementia with Lewy bodies. J Neurol 2010;257:359-66.

24. Irwin DJ, Grossman M, Weintraub D, et al. Neuropathological and genetic correlates of survival and dementia onset in synucleinopathies: a retrospective analysis. Lancet Neurol 2017;16:55-65.

25. Robinson L, Tang E, Taylor JP. Dementia: timely diagnosis and early intervention. BMJ 2015;350:h3029.

26. Cambridgeshire and Peterborough NHS Foundation Trust. Operational plan document for 2016-17, 2016. http://www.cpft. nhs.uk/Downloads/DVD-Documents/Publications/Annual-reports/ Operational\%20Plan\%202016\%2017\%20final.pdf 